\title{
New results on the energy of integral circulant graphs
}

\author{
Aleksandar Ilić \\ Faculty of Sciences and Mathematics, Višegradska 33, 18000 Niš, Serbia \\ e-mail: aleksandari@gmail.com \\ Milan Bašić \\ Faculty of Sciences and Mathematics, Višegradska 33, 18000 Nǐ̌, Serbia \\ e-mail: basic_milan@yahoo.com
}

May 29, 2018

\begin{abstract}
Circulant graphs are an important class of interconnection networks in parallel and distributed computing. Integral circulant graphs play an important role in modeling quantum spin networks supporting the perfect state transfer as well. The integral circulant graph $\operatorname{ICG}_{n}(D)$ has the vertex set $Z_{n}=\{0,1,2, \ldots, n-1\}$ and vertices $a$ and $b$ are adjacent if $\operatorname{gcd}(a-b, n) \in D$, where $D \subseteq\{d$ : $d \mid n, 1 \leq d<n\}$. These graphs are highly symmetric, have integral spectra and some remarkable properties connecting chemical graph theory and number theory. The energy of a graph was first defined by Gutman, as the sum of the absolute values of the eigenvalues of the adjacency matrix. Recently, there was a vast research for the pairs and families of non-cospectral graphs having equal energies. Following [R. B. Bapat, S. Pati, Energy of a graph is never an odd integer, Bull. Kerala Math. Assoc. 1 (2004) 129-132.], we characterize the energy of integral circulant graph modulo 4. Furthermore, we establish some general closed form expressions for the energy of integral circulant graphs and generalize some results from [A. Ilić, The energy of unitary Cayley graphs, Linear Algebra Appl. 431 (2009), 1881-1889.]. We close the paper by proposing some open problems and characterizing extremal graphs with minimal energy among integral circulant graphs with $n$ vertices, provided $n$ is even.
\end{abstract}

Key words: integral circulant graphs; graph energy; eigenvalues; cospectral graphs.

AMS Classifications: 05C50.

\section{Introduction}

Circulant graphs are Cayley graphs over a cyclic group. The interest of circulant graphs in graph theory and applications has grown during the last two decades, they appeared in coding theory, VLSI design, Ramsey theory and other areas. Recently there is vast research on the interconnection schemes based on circulant topology - circulant graphs represent an important class of interconnection networks in parallel and distributed computing (see [21]). Integral circulant graphs are also highly symmetric and have some remarkable properties between connecting graph theory and number theory.

In quantum communication scenario, circulant graphs is used in the problem of arranging $N$ interacting qubits in a quantum spin network based on a circulant topology to obtain good communication between them. In general, quantum spin system can be defined as a collection of qubits on a graph, whose dynamics is governed by a suitable Hamiltonian, without external control on the system. Different classes of graphs were examined for the purpose of perfect transferring the states of the systems. Since circulant graphs are mirror symmetric, they represent good candidates for the property of periodicity and thus integrality [12, which further implies that integral circulant graphs would be potential candidates for modeling the quantum spin networks that permit perfect state transfer [1, 2, 3, 14, 33]. 
These properties are primarily related to the spectra of these graphs. Indeed, the eigenvalues of the graphs are indexed in palindromic order $\left(\lambda_{i}=\lambda_{n-i}\right)$ and can be represented by Ramanujan's sums.

Bašić [7, 8] established a condition under which integral circulant graphs have perfect state transfer and gave complete characterization these graphs. It turned out that the degree of 2 must be equal in a prime factorization of the difference of successive eigenvalues. Furthermore, exactly one of the divisors $n / 4$ or $n / 2$ have to belong to the divisor set $D$ for any integral circulant graph $\operatorname{ICG}_{n}(D)$ having perfect state transfer. In this paper we continue with studying parameters of integral circulant graphs like energy, having in mind application in chemical graph theory. We actually focus on characterization of the energy of integral circulant graphs $\operatorname{ICG}_{n}(D)$ modulo 4 , where the divisor $n / 2$ and eigenvalue $\lambda_{n / 2}$ play important role. During this task, some interesting properties of the eigenvalues modulo 2 are also used.

Saxena, Severini and Shraplinski [33] studied some parameters of integral circulant graphs as the bounds for the number of vertices and the diameter, bipartiteness and perfect state transfer. The present authors in [6, 23] calculated the clique and chromatic number of integral circulant graphs with exactly one and two divisors, and also disproved posed conjecture that the order of $\operatorname{ICG}_{n}(D)$ is divisible by the clique number. Klotz and Sander [26] determined the diameter, clique number, chromatic number and eigenvalues of the unitary Cayley graphs. The latter group of authors proposed a generalization of unitary Cayley graphs named gcd-graphs and proved that they have to be integral.

Let $A$ be the adjacency matrix of a simple graph $G$, and $\lambda_{1}, \lambda_{2}, \ldots, \lambda_{n}$ be the eigenvalues of the graph $G$. The energy of $G$ is defined as the sum of absolute values of its eigenvalues [15, 16, 19]

$$
E(G)=\sum_{i=1}^{n}\left|\lambda_{i}\right| .
$$

The concept of graph energy arose in chemistry where certain numerical quantities, such as the heat of formation of a hydrocarbon, are related to total $\pi$-electron energy that can be calculated as the energy of an appropriate molecular graph.

The graph $G$ is said to be hyperenergetic if its energy exceeds the energy of the complete graph $K_{n}$, or equivalently if $E(G)>2 n-2$. This concept was introduced first by Gutman and afterwards has been studied intensively in the literature [4, 9, 17, 35. Hyperenergetic graphs are important because molecular graphs with maximum energy pertain to maximality stable $\pi$-electron systems. In [22] and [31, the authors calculated the energy of unitary Cayley graphs and complement of unitary Cayley graphs, and establish the necessary and sufficient conditions for $\mathrm{ICG}_{n}$ to be hyperenergetic. There was a vast research for the pairs and families of non-cospectral graphs having equal energy [10, 11, 24, 25, 27, 28, 30, 36].

In 2004 Bapat and Pati [5] proved an interesting simple result - the energy of a graph cannot be an odd integer. Pirzada and Gutman [29] generalized this result and proved the following

Theorem 1.1 Let $r$ and $s$ be integers such that $r \geq 1$ and $0 \leq s \leq r-1$. Let $q$ be an odd integer. Then $E(G)$ cannot be of the form $\left(2^{s} q\right)^{1 / r}$.

For more information about the closed forms of the graph energy we refer the reader to [32].

In this paper we go a step further and characterize the energy of integral circulant graph modulo 4.

The paper is organized as follows. In Section 2 we give some preliminary results regarding eigenvalues of integral circulant graphs. In Section 3 we characterize the energy of integral circulant graph modulo 4, while in Section 4 we generalized formulas for the energy of integral circulant graphs from 22. In Section 5, some larger families of graphs with equal energy are presented and further we support conjecture proposed by So [34, that two graphs $\operatorname{ICG}_{n}\left(D_{1}\right)$ and $\operatorname{ICG}_{n}\left(D_{2}\right)$ are cospectral if and only if $D_{1}=D_{2}$. In concluding remarks we propose some open problems and characterize extremal graphs with minimal energy among integral circulant graphs with $n$ vertices, provided $n$ is even. 


\section{Preliminaries}

Let us recall that for a positive integer $n$ and subset $S \subseteq\{0,1,2, \ldots, n-1\}$, the circulant graph $G(n, S)$ is the graph with $n$ vertices, labeled with integers modulo $n$, such that each vertex $i$ is adjacent to $|S|$ other vertices $\{i+s(\bmod n) \mid s \in S\}$. The set $S$ is called a symbol of $G(n, S)$. As we will consider only undirected graphs without loops, we assume that $0 \notin S$ and, $s \in S$ if and only if $n-s \in S$, and therefore the vertex $i$ is adjacent to vertices $i \pm s(\bmod n)$ for each $s \in S$.

Recently, So [34 has characterized circulant graphs with integral eigenvalues-integral circulant graphs. Let

$$
G_{n}(d)=\{k \mid \operatorname{gcd}(k, n)=d, 1 \leq k<n\}
$$

be the set of all positive integers less than $n$ having the same greatest common divisor $d$ with $n$. Let $D_{n}$ be the set of positive divisors $d$ of $n$, with $d \leq \frac{n}{2}$.

Theorem 2.1 A circulant graph $G(n, S)$ is integral if and only if

$$
S=\bigcup_{d \in D} G_{n}(d)
$$

for some set of divisors $D \subseteq D_{n}$.

We denote them by $\operatorname{ICG}_{n}(D)$ and in some recent papers integral circulant graphs are also known as gcd-graphs $([6,26])$.

Let $\Gamma$ be a multiplicative group with identity $e$. For $S \subset \Gamma, e \notin S$ and $S^{-1}=\left\{s^{-1} \mid s \in S\right\}=S$, the Cayley graph $X=\operatorname{Cay}(\Gamma, S)$ is the undirected graph having vertex set $V(X)=\Gamma$ and edge set $E(X)=\left\{\{a, b\} \mid a b^{-1} \in S\right\}$. For a positive integer $n>1$ the unitary Cayley graph $X_{n}=\operatorname{Cay}\left(Z_{n}, U_{n}\right)$ is defined by the additive group of the ring $Z_{n}$ of integers modulo $n$ and the multiplicative group $U_{n}=Z_{n}^{*}$ of its invertible elements.

By Theorem 2.1 we obtain that integral circulant graphs are Cayley graphs of the additive group of $Z_{n}$ with respect to the Cayley set $S=\bigcup_{d \in D} G_{n}(d)$. From Corollary 4.2 in [21], the graph $\operatorname{ICG}_{n}(D)$ is connected if and only if $\operatorname{gcd}\left(d_{1}, d_{2}, \ldots, d_{k}\right)=1$.

Let $A$ be a circulant matrix. The entries $a_{0}, a_{1}, \ldots, a_{n-1}$ of the first row of the circulant matrix $A$ generate the entries of the other rows by a cyclic shift (for more details see [13]). There is an explicit formula for the eigenvalues $\lambda_{k}, 0 \leqslant k \leqslant n-1$, of a circulant matrix $A$. Define the polynomial $P_{n}(z)$ by the entries of the first row of $A$,

$$
P_{n}(z)=\sum_{i=0}^{n-1} a_{i} \cdot z^{i}
$$

The eigenvalues of $A$ are given by

$$
\lambda_{j}=P_{n}\left(\omega^{j}\right)=\sum_{i=0}^{n-1} a_{i} \cdot \omega^{j i}, \quad 0 \leqslant j \leqslant n-1,
$$

where $\omega=\exp (12 \pi / n)$ is the $n$-th root of unity. Ramanujan's sum [38, usually denoted $c(k, n)$, is a function of two positive integer variables $n$ and $k$ defined by the formula

$$
c(k, n)=\sum_{\substack{a=1 \\ \operatorname{gcd}(a, n)=1}}^{n} e^{\frac{2 \pi i}{n} \cdot a k}=\sum_{\substack{a=1 \\ \operatorname{gcd}(a, n)=1}}^{n} \omega_{n}^{a k},
$$

where $\omega_{n}$ denotes a complex primitive $n$-th root of unity. These sums take only integral values,

$$
c(k, n)=\mu\left(t_{n, k}\right) \cdot \frac{\varphi(n)}{\varphi\left(t_{n, k}\right)} \quad \text { where } \quad t_{n, k}=\frac{n}{\operatorname{gcd}(k, n)},
$$


and $\mu$ denotes the Möbious function. In [26] it was proven that gcd-graphs (the same term as integral circulant graphs $\operatorname{ICG}_{n}(D)$ ) have integral spectrum,

$$
\lambda_{k}=\sum_{d \in D} c\left(k, \frac{n}{d}\right), \quad 0 \leqslant k \leqslant n-1 .
$$

Using the well-known summation [20]

$$
s(k, n)=\sum_{i=0}^{n-1} \omega_{n}^{i k}=\left\{\begin{array}{lll}
0 & \text { if } & n \nmid k \\
n & \text { if } & n \mid k
\end{array},\right.
$$

we get that

$$
\sum_{k=0}^{n-1} c(k, n)=0
$$

For even $n$ it follows

$$
\begin{aligned}
\sum_{k=0}^{n / 2-1} c(k, n) & =\sum_{\substack{a=1 \\
\operatorname{gcd}(a, n)=1}}^{n} \sum_{k=0}^{n / 2-1} \omega_{n}^{a k}=\sum_{\substack{a=1 \\
\operatorname{gcd}(a, n)=1}}^{n / 2}\left(\sum_{k=0}^{n / 2-1} \omega_{n}^{a k}+\omega_{n}^{(n-a) k}\right) \\
& =\sum_{\substack{a=1 \\
\operatorname{gcd}(a, n)=1}}^{n / 2}\left(\omega_{n}^{a n}-\omega_{n}^{a n / 2}+\sum_{k=0}^{n-1} \omega_{n}^{a k}\right)=\sum_{\substack{a=1 \\
\operatorname{gcd}(a, n)=1}}^{n / 2}(1+1) \\
& =\varphi(n) .
\end{aligned}
$$

Similarly, for odd $n$ it follows

$$
\sum_{k=0}^{(n-1) / 2} c(k, n)=\frac{\varphi(n)}{2} .
$$

It also follows that if $k \equiv k^{\prime}(\bmod n)$ then $c(k, n)=c\left(k^{\prime}, n\right)$.

Throughout the paper, we let $n=p_{1}^{\alpha_{1}} p_{2}^{\alpha_{2}} \cdot \ldots \cdot p_{k}^{\alpha_{k}}$, where $p_{1}<p_{2}<\ldots<p_{k}$ are distinct primes, and $\alpha_{i} \geq 1$.

\section{The energy of integral circulant graphs modulo 4}

Note that for arbitrary divisor $d$ and $1 \leq i \leq n-1$, it holds

$$
t_{n / d, i}=\frac{n / d}{\operatorname{gcd}(n / d, i)}=\frac{n}{\operatorname{gcd}(n, i d)}
$$

and

$$
t_{n / d, n-i}=\frac{n / d}{\operatorname{gcd}(n / d, n-i)}=\frac{n}{\operatorname{gcd}(n, n d-i d)} .
$$

Since $\operatorname{gcd}(n, i d)=\operatorname{gcd}(n, n d-i d)$, we have $t_{n / d, i}=t_{n / d, n-i}$. Finally,

$$
c(i, n / d)=\mu\left(t_{n / d, i}\right) \frac{\varphi(n / d)}{\varphi\left(t_{n / d, i}\right)}=\mu\left(t_{n / d, n-i}\right) \frac{\varphi(n / d)}{\varphi\left(t_{n / d, n-i}\right)}=c(n-i, n / d),
$$

for each $1 \leq i \leq n-1$. Therefore we have the following assertion.

Lemma 3.1 Let $\operatorname{ICG}_{n}(D)$ be an arbitrary integral circulant graph. Then for each $1 \leq i \leq n-1$, the eigenvalues $\lambda_{i}$ and $\lambda_{n-i}$ of $\mathrm{ICG}_{n}(D)$ are equal. 
For $i=0$ we have

$$
\lambda_{0}=\sum_{d \in D} \varphi(n / d)
$$

while for $n$ even and $i=n / 2$ we have

$$
\lambda_{n / 2}=\sum_{d \in D}(-1)^{d} \varphi(n / d)
$$

\subsection{Energy modulo 4 for $n$ odd}

According to Lemma 3.1, the energy of $G \cong \operatorname{ICG}_{n}(D)$ is equal to

$$
E(G)=\lambda_{0}+2 \sum_{i=1}^{(n-1) / 2}\left|\lambda_{i}\right|
$$

Since $x \equiv|x|(\bmod 2)$, in order to characterize $E(G)$ modulo 4 we consider the parity of the following sum

$$
\frac{E(G)}{2} \equiv \sum_{d \in D} \frac{\varphi(n / d)}{2}+\sum_{i=1}^{(n-1) / 2} \sum_{d \in D} c(i, n / d) \quad(\bmod 2) .
$$

Since $n / d>2$, it follows that $\varphi(n / d)$ is even. After exchanging the order of the summation we have

$$
\frac{E(G)}{2} \equiv \sum_{d \in D} \frac{\varphi(n / d)}{2}+\sum_{d \in D} \sum_{i=1}^{(n-1) / 2} c(i, n / d) \quad(\bmod 2)
$$

By relation (3), we get that for every $k$ it holds that

$$
\sum_{i=k}^{k+n-1} c(i, n)=0
$$

Theorem 3.2 For odd $n$, the energy of $\operatorname{ICG}_{n}(D)$ is divisible by four.

Proof: Using the following relation $\frac{n-1}{2}=\frac{n}{d} \cdot \frac{d-1}{2}+\frac{n-d}{2 d}$, the formula for graph energy (6) now becomes

$$
\frac{E(G)}{2} \equiv \sum_{d \in D} \frac{\varphi(n / d)}{2}+\sum_{d \in D}\left(\sum_{l=0}^{\frac{d-1}{2}-1} \sum_{i=l \frac{n}{d}+1}^{(l+1) \frac{n}{d}} c(i, n / d)+\sum_{i=\frac{n(d-1)}{2 d}+1}^{\frac{n-1}{2}} c(i, n / d)\right) \quad(\bmod 2) .
$$

Next we get

$$
\frac{E(G)}{2} \equiv \sum_{d \in D} \frac{\varphi(n / d)}{2}+\sum_{i=1}^{(n / d-1) / 2} c(i, n / d) \quad(\bmod 2)
$$

and using relation (5), we get that

$$
\frac{E(G)}{2} \equiv \sum_{d \in D} \frac{\varphi(n / d)}{2}+\frac{\varphi(n / d)}{2}-\varphi(n / d) \equiv 0 \quad(\bmod 2) .
$$

This implies that $4 \mid E(G)$. 


\subsection{Energy modulo 4 for $n$ even}

According to Lemma 3.1, the energy of $G \cong \operatorname{ICG}_{n}(D)$ is equal to

$$
E(G)=\left|\lambda_{0}\right|+\left|\lambda_{n / 2}\right|+2 \sum_{i=1}^{n / 2-1}\left|\lambda_{i}\right| .
$$

Using the same reasoning as in the previous subsection, we get that $\lambda_{0}$ and $\lambda_{n / 2}$ are of the same parity,

$$
\left|\lambda_{0}\right|+\left|\lambda_{n / 2}\right|=\sum_{d \in D} \varphi(n / d)+\left|\sum_{d \in D}(-1)^{d} \varphi(n / d)\right| .
$$

Also,

$$
\begin{aligned}
S & =\frac{1}{2} \cdot\left(\left|\lambda_{0}\right|+\left|\lambda_{n / 2}\right|\right)=\sum_{d \in D} \frac{\varphi(n / d)}{2}+\left|\sum_{d \in D}(-1)^{d} \frac{\varphi(n / d)}{2}\right| \\
& =\left\{\begin{array}{ll}
\sum_{d \in D, d \text { even }} \varphi(n / d), & \text { if } \lambda_{n / 2}>0 \\
\sum_{d \in D, d \text { odd }} \varphi(n / d), & \text { if } \lambda_{n / 2}<0
\end{array} .\right.
\end{aligned}
$$

If $\frac{n}{2} \notin D$, then $2 \mid \varphi(n / d)$ and $S \equiv 0(\bmod 2)$; otherwise we conclude that

$$
S \equiv\left\{\begin{array}{lll}
0, & \text { if } \lambda_{n / 2}>0 \text { and } 4 \nmid n, \text { or } \lambda_{n / 2}<0 \text { and } 4 \mid n & (\bmod 2) \\
1, & \text { if } \lambda_{n / 2}>0 \text { and } 4 \mid n, \text { or } \lambda_{n / 2}<0 \text { and } 4 \nmid n & (\bmod 2)
\end{array} .\right.
$$

Therefore

$$
\frac{E(G)}{2} \equiv S+\sum_{d \in D} \sum_{i=1}^{n / 2-1} c(i, n / d) \quad(\bmod 2)
$$

Theorem 3.3 For even $n$, the energy of $\operatorname{ICG}_{n}(D)$ is not divisible by four if and only if $\frac{n}{2} \notin D$ and $\lambda_{n / 2}$ is negative.

Proof: If $d$ is even, we have $\frac{n}{2}-1=\frac{d}{2} \cdot \frac{n}{d}-1$. Since $c(0, n / d)=\varphi(n / d)$, it follows

$$
\begin{aligned}
\sum_{i=1}^{n / 2-1} c(i, n / d) & =-c(0, n / d)+\sum_{k=1}^{d / 2} \sum_{i=(k-1) \cdot n / d}^{k \cdot n / d-1} c(i, n / d) \\
& =-\varphi(n / d)+\frac{d}{2} \cdot \sum_{i=0}^{n / d-1} c(i, n / d) \\
& =-\varphi(n / d) .
\end{aligned}
$$

If $d$ is odd, we have $\frac{n}{2}-1=\frac{d-1}{2} \cdot \frac{n}{d}+\frac{1}{2} \cdot \frac{n}{d}-1$. Similarly, using the relation (4), it follows

$$
\begin{aligned}
\sum_{i=1}^{n / 2-1} c(i, n / d) & =-c(0, n / d)+\sum_{k=1}^{(d-1) / 2} \sum_{i=(k-1) \cdot n / d}^{k \cdot n / d-1} c(i, n / d)+\sum_{i=((d-1) / 2) \cdot n / d}^{n / 2-1} c(i, n / d) \\
& =-\varphi(n / d)+\frac{d-1}{2} \cdot \sum_{i=0}^{n / d-1} c(i, n / d)+\sum_{i=0}^{n /(2 d)-1} c(i, n / d) \\
& =-\varphi(n / d)+\varphi(n / d)=0 .
\end{aligned}
$$

For $\frac{n}{2} \notin D$, we have that $S \equiv 0(\bmod 2)$ and $4 \mid E(G)$. 
For $\frac{n}{2} \in D$, by combining above cases we have

$$
\sum_{d \in D} \sum_{i=1}^{n / 2-1} c(i, n / d) \equiv \frac{1+(-1)^{n / 2}}{2}(\bmod 2)
$$

For $\lambda_{n / 2}>0$, it follows

$$
\frac{E(G)}{2} \equiv S+\frac{1+(-1)^{n / 2}}{2} \equiv \frac{1+(-1)^{n / 2}}{2}+\frac{1+(-1)^{n / 2}}{2} \equiv 0 \quad(\bmod 2)
$$

while for $\lambda_{n / 2}<0$, we have

$$
\frac{E(G)}{2} \equiv S+\frac{1+(-1)^{n / 2}}{2} \equiv \frac{1-(-1)^{n / 2}}{2}+\frac{1+(-1)^{n / 2}}{2} \equiv 1 \quad(\bmod 2)
$$

This completes the proof.

\section{The energy of some classes of integral circulant graphs}

Here we generalize results from [22].

Theorem 4.1 Let $n \geq 4$ be an arbitrary integer. Then the energy of the integral circulant graph $X_{n}\left(1, p^{\gamma}\right)$ for $\gamma \geq 1$ is given by

$$
E\left(X_{n}\left(1, p^{\gamma}\right)\right)=\left\{\begin{aligned}
2^{k-1}(\varphi(n)+\varphi(n / p)), & p \| n \\
2^{k-1}\left(2 \varphi(n)+\left(p^{\gamma}-2 p+2\right) \varphi(n / p)\right), & p^{\gamma} \| n, \gamma \geq 2 \\
2^{k}\left(\varphi(n)+\left(p^{\gamma}-p+1\right) \varphi(n / p)\right), & p^{\gamma} \not n
\end{aligned}\right.
$$

Proof: Let $p=p_{s}$ and $\gamma=\gamma_{s}$, where $1 \leq s \leq k$. Let $j=p_{1}{ }^{\beta_{1}} p_{2}^{\beta_{2}} \cdot \ldots \cdot p_{k}^{\beta_{k}} \cdot J$ be a representation of an arbitrary index $0 \leq j \leq n-1$, where $\operatorname{gcd}(J, n)=1$. The $j$-th eigenvalue of $X_{n}\left(1, p_{s}^{\gamma_{s}}\right)$ is given by

$$
\lambda_{j}=c(j, n)+c\left(j, n / p_{s}^{\gamma_{s}}\right) .
$$

Suppose that there exists a prime number $p_{i} \mid j$ for some $i \neq s$ such $\beta_{i} \leq \alpha_{i}-2$. This implies that $p_{i}^{2} \mid t_{n, j}$ and $p_{i}^{2} \mid t_{n / p_{s}^{\gamma_{s}}, j}$. Furthermore, we have $\mu\left(t_{n, j}\right)=\mu\left(t_{n / p_{s}^{\gamma_{s}}, j}\right)=0$ and thus $\lambda_{j}=0$.

If $\beta_{s} \leq \alpha_{s}-\gamma_{s}-1$ then $p_{s}^{3} \mid t_{n, j}$ and $p_{s}^{2} \mid t_{n / p_{s}^{\gamma_{s}}, j}$. Similarly, we conclude that $\lambda_{j}=0$.

For an arbitrary index $j$, define the set $P=\left\{1 \leq i \leq k \mid i \neq s, \beta_{i}=\alpha_{i}-1\right\}$.

Let $J_{l}=\left\{0 \leq j \leq n-1 \mid \beta_{s}=\alpha_{s}-l, \alpha_{i}-1 \leq \beta_{i} \leq \alpha_{i}\right.$ for $\left.i \neq s\right\}$, for $0 \leq l \leq \gamma_{s}+1$.

Case 1. For $l=0$ and $j \in J_{0}$ we have

$$
t_{n, j}=\frac{n}{\operatorname{gcd}(j, n)}=\frac{p_{1}^{\alpha_{1}} p_{2}^{\alpha_{2}} \cdots p_{s}^{\alpha_{s}} \cdots p_{k}^{\alpha_{k}}}{p_{1}^{\beta_{1}} p_{2}^{\beta_{2}} \cdots p_{s}^{\alpha_{s}} \cdots p_{k}^{\beta_{k}}}=\prod_{i \in P} p_{i} .
$$

On the other hand, it follows

$$
t_{n / p_{s}^{\gamma_{s}}, j}=\frac{n / p_{s}^{\gamma_{s}}}{\operatorname{gcd}\left(j, n / p_{s}^{\gamma_{s}}\right)}=\frac{p_{1}^{\alpha_{1}} p_{2}^{\alpha_{2}} \cdots p_{s}^{\alpha_{s}-\gamma_{s}} \cdots p_{k}^{\alpha_{k}}}{p_{1}^{\beta_{1}} p_{2}^{\beta_{2}} \cdots p_{s}^{\alpha_{s}-\gamma_{s}} \cdots p_{k}^{\beta_{k}}}=\prod_{i \in P} p_{i}
$$

The $j$-th eigenvalue is given by

$$
\lambda_{j}=c(j, n)+c\left(j, n / p_{s}^{\gamma_{s}}\right)=(-1)^{|P|} \frac{\varphi(n)}{\varphi\left(\prod_{i \in P} p_{i}\right)}+(-1)^{|P|} \frac{\varphi\left(n / p_{s}^{\gamma_{s}}\right)}{\varphi\left(\prod_{i \in P} p_{i}\right)}=(-1)^{|P|} \frac{\varphi(n)+\varphi\left(n / p_{s}^{\gamma_{s}}\right)}{\varphi\left(\prod_{i \in P} p_{i}\right)}
$$


The number of indices $j \in J_{0}$ with the same set $P$ is equal to the number of $J$ such that

$$
\operatorname{gcd}\left(J, \frac{n}{p_{1}^{\beta_{1}} p_{2}^{\beta_{2}} \cdots p_{s}^{\alpha_{s}} \cdots p_{k}^{\beta_{k}}}\right)=1 .
$$

The last equation implies that the number of such indices is equal to the Euler's totient function

$$
\varphi\left(\frac{n}{p_{1}^{\beta_{1}} p_{2}^{\beta_{2}} \cdots p_{s}^{\alpha_{s}} \cdots p_{k}^{\beta_{k}}}\right)=\varphi\left(\prod_{i \in P} p_{i}\right) .
$$

Case 2. Let $l=1$ and for $j \in J_{1}$ we similarly obtain $t_{n, j}=p_{s} \prod_{i \in P} p_{i}$ and $t_{n / p_{s}^{\gamma_{s}}, j}=\prod_{i \in P} p_{i}$. Therefore, the $j$-th eigenvalue is given by

$$
\lambda_{j}=(-1)^{|P|+1} \frac{\varphi(n)}{\varphi\left(p_{s} \prod_{i \in P} p_{i}\right)}+(-1)^{|P|} \frac{\varphi\left(n / p_{s}^{\gamma_{s}}\right)}{\varphi\left(\prod_{i \in P} p_{i}\right)}=(-1)^{|P|} \frac{\left(-\varphi(n)+\left(p_{s}-1\right) \varphi\left(n / p_{s}^{\gamma_{s}}\right)\right)}{\left(p_{s}-1\right) \varphi\left(\prod_{i \in P} p_{i}\right)} .
$$

The number of indices $j \in J_{1}$ with the same set $P$ is equal to

$$
\varphi\left(\frac{n}{p_{1}^{\beta_{1}} p_{2}^{\beta_{2}} \cdots p_{s}^{\alpha_{s}-1} \cdots p_{k}^{\beta_{k}}}\right)=\varphi\left(p_{s} \prod_{i \in P} p_{i}\right)=\left(p_{s}-1\right) \varphi\left(\prod_{i \in P} p_{i}\right) .
$$

Case 3. For $2 \leq l \leq \gamma_{s}$ and $j \in J_{l}$ we obtain $p_{s}^{\alpha_{s}-\gamma_{s}-\min \left(\alpha_{s}-l, \alpha_{s}-\gamma_{s}\right)} \| t_{n / p_{s}^{\gamma_{s}}, j}$ which implies that $p_{s} \nmid t_{n / p_{s}^{\gamma_{s}}, j}$ and $t_{n / p_{s}^{\gamma_{s}}, j}=\prod_{i \in P} p_{i}$. Since $p_{s}^{l} \mid t_{n, j}$ and $l \geq 2$ it holds that $\mu\left(t_{n, j}\right)=0$. Therefore, the $j$-th eigenvalue is given by

$$
\lambda_{j}=(-1)^{|P|} \frac{\varphi\left(n / p_{s}^{\gamma_{s}}\right)}{\varphi\left(\prod_{i \in P} p_{i}\right)}=(-1)^{|P|} \frac{\varphi\left(n / p_{s}^{\gamma_{s}}\right)}{\varphi\left(\prod_{i \in P} p_{i}\right)} .
$$

The number of indices $j \in J_{l}$ with the same set $P$ is equal to

$$
\varphi\left(\frac{n}{p_{1}^{\beta_{1}} p_{2}^{\beta_{2}} \cdots p_{s}^{\alpha_{s}-l} \cdots p_{k}^{\beta_{k}}}\right)=\varphi\left(p_{s}^{l} \prod_{i \in P} p_{i}\right)=p_{s}^{l-1}\left(p_{s}-1\right) \varphi\left(\prod_{i \in P} p_{i}\right) .
$$

Case 4. For $l=\gamma_{s}+1$ and $j \in J_{\gamma_{s}+1}$ we obtain $p_{s}^{\gamma_{s}+1} \| t_{n, j}$ and $c(j, n)=\mu\left(t_{n, j}\right)=0$. Also, it holds that $p_{s}^{\alpha_{s}-\gamma_{s}-\min \left(\alpha_{s}-\gamma_{s}-1, \alpha_{s}-\gamma_{s}\right)} \| t_{n / p_{s}^{\gamma_{s}}, j}$ which yields that $p_{s} \| t_{n / p_{s}^{\gamma_{s}}, j}$. Therefore, the $j$-th eigenvalue is given by

$$
\lambda_{j}=(-1)^{|P|+1} \frac{\varphi\left(n / p_{s}^{\gamma_{s}}\right)}{\varphi\left(p_{s} \prod_{i \in P} p_{i}\right)}=(-1)^{|P|+1} \frac{\varphi\left(n / p_{s}^{\gamma_{s}}\right)}{\varphi\left(p_{s} \prod_{i \in P} p_{i}\right)} .
$$

The number of indices $j \in J_{l}$ with the same set $P$ is equal to

$$
\varphi\left(\frac{n}{p_{1}^{\beta_{1}} p_{2}^{\beta_{2}} \cdots p_{s}^{\alpha_{s}-\gamma_{s}-1} \cdots p_{k}^{\beta_{k}}}\right)=\varphi\left(p_{s}^{\gamma_{s}+1} \prod_{i \in P} p_{i}\right)=p_{s}^{\gamma_{s}}\left(p_{s}-1\right) \varphi\left(\prod_{i \in P} p_{i}\right)
$$

After all mention cases, the energy of $X_{n}\left(1, p_{s}^{\gamma_{s}}\right)$ is given by

$$
\begin{aligned}
E\left(X_{n}\left(1, p_{s}^{\gamma_{s}}\right)\right)= & \sum_{j=0}^{n-1}\left|\lambda_{j}\right| \\
= & \sum_{P \subseteq\{1,2, \ldots, k\} \backslash\{s\}}\left(\frac{\varphi(n)+\varphi\left(n / p_{s}^{\gamma_{s}}\right)}{\varphi\left(\prod_{i \in P} p_{i}\right)} \cdot \varphi\left(\prod_{i \in P} p_{i}\right)\right. \\
& +\frac{\varphi(n)-\left(p_{s}-1\right) \varphi\left(n / p_{s}^{\gamma_{s}}\right)}{\left(p_{s}-1\right) \varphi\left(\prod_{i \in P} p_{i}\right)} \cdot\left(p_{s}-1\right) \varphi\left(\prod_{i \in P} p_{i}\right) \\
& +\sum_{l=2}^{\gamma_{s}} \frac{\varphi\left(n / p_{s}^{\gamma_{s}}\right)}{\varphi\left(\prod_{i \in P} p_{i}\right)} \cdot p_{s}^{l-1}\left(p_{s}-1\right) \varphi\left(\prod_{i \in P} p_{i}\right) \\
& \left.+\frac{\varphi\left(n / p_{s}^{\gamma_{s}}\right)}{\left(p_{s}-1\right) \varphi\left(\prod_{i \in P} p_{i}\right)} \cdot p_{s}^{\gamma_{s}}\left(p_{s}-1\right) \varphi\left(\prod_{i \in P} p_{i}\right)\right)
\end{aligned}
$$


If $\alpha_{s}=1$ then $J_{l}=\emptyset$ for $l \geq 2$ and $\gamma_{s}=1$. Since the Euler totient function is multiplicative, for $\alpha_{s}=1$ we have $\varphi(n)=\left(p_{s}-1\right) \varphi\left(n / p_{s}\right)$. Thus, the relation (10) becomes

$$
E\left(X_{n}\left(1, p_{s}\right)\right)=2^{k-1} \cdot\left(\varphi(n)+\varphi\left(n / p_{s}\right)+\varphi(n)-\left(p_{s}-1\right) \varphi\left(n / p_{s}\right)\right)=2^{k-1}\left(\varphi(n)+\varphi\left(n / p_{s}\right)\right) .
$$

If $\alpha_{s}=\gamma_{s} \geq 2$ then $J_{\gamma_{s+1}}=\emptyset$ since $\beta_{s}=\alpha_{s}-\gamma_{s}-1<0$ is not defined. Thus, the relation (10) is reduced to the first three summands as follows

$$
\begin{aligned}
E\left(X_{n}\left(1, p_{s}^{\gamma_{s}}\right)\right) & =2^{k-1} \cdot\left(\left(\varphi(n)+\varphi\left(n / p_{s}\right)\right)+\left(\varphi(n)-\left(p_{s}-1\right) \varphi\left(n / p_{s}\right)\right)+\left(p_{s}-1\right) \varphi\left(n / p_{s}^{\gamma_{s}}\right) \sum_{l=2}^{\gamma_{s}} p_{s}^{l-1}\right) \\
& =2^{k-1} \cdot\left(2 \varphi(n)+\left(p_{s}-2\right) \varphi\left(n / p_{s}^{\gamma_{s}}\right)+p_{s}\left(p_{s}^{\gamma_{s}-1}-1\right) \varphi\left(n / p_{s}^{\gamma_{s}}\right)\right) \\
& =2^{k-1}\left(2 \varphi(n)+\left(p_{s}^{\gamma_{s}}-2 p_{s}+2\right) \varphi\left(n / p_{s}\right)\right) .
\end{aligned}
$$

If $\alpha_{s}>\gamma_{s} \geq 2$ the formula (10) is composed of four summands, thus we have

$$
\begin{aligned}
E\left(X_{n}\left(1, p_{s}^{\gamma_{s}}\right)\right) & =2^{k-1}\left(2 \varphi(n)+\left(p_{s}^{\gamma_{s}}-2 p_{s}+2\right) \varphi\left(n / p_{s}\right)+p_{s}^{\gamma_{s}} \varphi\left(n / p_{s}^{\gamma_{s}}\right)\right) \\
& =2^{k}\left(\varphi(n)+\left(p_{s}^{\gamma_{s}}-p_{s}+1\right) \varphi\left(n / p_{s}\right)\right) .
\end{aligned}
$$

This completes the proof.

Theorem 4.2 Let $n \geq 4$ be an arbitrary integer. Then the energy of the integral circulant graph $X_{n}(p, q)$ for $p=p_{s}$ and $q=p_{t}$, where $1 \leq s<t \leq k$, is given by

$$
E\left(X_{n}(p, q)\right)=\left\{\begin{aligned}
2^{k} \varphi(n), & p\|n q\| n \\
3 \cdot 2^{k-1} \varphi(n), & 2 \| n q^{2} \mid n \\
2^{k-1}\left(2 \varphi(n)+\varphi\left(n / p_{t}\right) \varphi\left(p_{t}\right)\right), & p \| n q^{2} \mid n p \neq 2 \\
2^{k-1}\left(2 \varphi(n)+\varphi\left(n / p_{s}\right) \varphi\left(p_{s}\right)\right), & p^{2} \mid n q \| n \\
2^{k-1}\left(2 \varphi(n)+\varphi\left(n / p_{s}\right) \varphi\left(p_{s}\right)+\varphi\left(n / p_{t}\right) \varphi\left(p_{t}\right)\right), & p^{2}\left|n q^{2}\right| n
\end{aligned}\right.
$$

Proof: Let $j=p_{1}{ }^{\beta_{1}} p_{2}^{\beta_{2}} \cdot \ldots \cdot p_{k}^{\beta_{k}} \cdot J$ be a representation of an arbitrary index $0 \leq j \leq n-1$, where $\operatorname{gcd}(J, n)=1$. The $j$-th eigenvalue of $X_{n}\left(p_{s}, p_{t}\right)$ is given by

$$
\lambda_{j}=c\left(j, n / p_{s}\right)+c\left(j, n / p_{t}\right) .
$$

Suppose that there exists prime number $p_{i} \mid j$ for some $i \neq s, t$ such $\beta_{i} \leq \alpha_{i}-2$. This implies that $p_{i}^{2} \mid t_{n / p_{s}, j}$ and $p_{i}^{2} \mid t_{n / p_{t}, j}$. Furthermore, we have $\mu\left(t_{n / p_{t}, j}\right)=\mu\left(t_{n / p_{s}, j}\right)=0$ and thus $\lambda_{j}=0$.

If $\beta_{s} \leq \alpha_{s}-3$ then $p_{s}^{3} \mid t_{n / p_{t}, j}$ and $p_{s}^{2} \mid t_{n / p_{s}, j}$. Similarly, we conclude that $\lambda_{j}=0$.

If $\beta_{t} \leq \alpha_{t}-3$ then $p_{t}^{3} \mid t_{n / p_{s}, j}$ and $p_{t}^{2} \mid t_{n / p_{t}, j}$. Similarly, we conclude that $\lambda_{j}=0$.

For an arbitrary index $j$, define the set $P=\left\{1 \leq i \leq k \mid i \neq s, t, \beta_{i}=\alpha_{i}-1\right\}$.

Let $J_{l_{1}, l_{2}}=\left\{0 \leq j \leq n-1 \mid \beta_{s}=\alpha_{s}-l_{1}, \beta_{t}=\alpha_{t}-l_{2}, \alpha_{i}-1 \leq \beta_{i} \leq \alpha_{i}\right.$ for $\left.i \neq s, t\right\}$.

For $j \in J_{l_{1}, l_{2}}$, where $0 \leq l_{1}, l_{2} \leq 2$, we have

$$
t_{n / p_{s}, j}=\frac{n / p_{s}}{g c d\left(j, n / p_{s}\right)}=p_{s}^{\alpha_{s}-1-\min \left(\alpha_{s}-1, \alpha_{s}-l_{1}\right)} p_{t}^{\alpha_{t}-\left(\alpha_{t}-l_{2}\right)} \prod_{i \in P} p_{i}=\left\{\begin{array}{cl}
p_{t}^{l_{2}} \prod_{i \in P} p_{i}, & 0 \leq l_{1} \leq 1 \\
p_{s} p_{t}^{l_{2}} \prod_{i \in P} p_{i}, & l_{1}=2
\end{array} .\right.
$$

Similarly it follows

$$
t_{n / p_{t}, j}=\left\{\begin{array}{cl}
p_{s}^{l_{1}} \prod_{i \in P} p_{i}, & 0 \leq l_{2} \leq 1 \\
p_{t} p_{s}^{l_{1}} \prod_{i \in P} p_{i}, & l_{2}=2
\end{array}\right.
$$


The number of indices $j \in J_{l_{1}, l_{2}}$ with the same set $P$ is equal to the number of $J$ such that

$$
\operatorname{gcd}\left(J, \frac{n}{p_{1}^{\beta_{1}} p_{2}^{\beta_{2}} \cdots p_{s}^{\alpha_{s}-l_{1}} \cdots p_{t}^{\alpha_{t}-l_{2}} \cdots p_{k}^{\beta_{k}}}\right)=1 .
$$

The last equation implies that the number of such indices is equal to

$$
\varphi\left(\frac{n}{p_{1}^{\beta_{1}} p_{2}^{\beta_{2}} \cdots p_{s}^{\alpha_{s}-l_{1}} \cdots p_{t}^{\alpha_{t}-l_{2}} \cdots p_{k}^{\beta_{k}}}\right)=\varphi\left(p_{s}^{l_{1}} p_{t}^{l_{2}} \prod_{i \in P} p_{i}\right) .
$$

Now, we distinguish four cases depending on the values of $l_{1}$ and $l_{2}$.

Case 1. $0 \leq l_{1}, l_{2} \leq 1$.

According to the relations (12) and (13) it follows $t_{n / p_{s}, j}=p_{t}^{l_{2}} \prod_{i \in P} p_{i}$ and $t_{n / p_{t}, j}=p_{s}^{l_{1}} \prod_{i \in P} p_{i}$ and therefore the $j$-th eigenvalue is given by

$$
\begin{aligned}
\lambda_{j}=c\left(j, n / p_{s}\right)+c\left(j, n / p_{t}\right) & =(-1)^{|P|+l_{2}} \frac{\varphi\left(n / p_{s}\right)}{\varphi\left(p_{t}^{l_{2}} \prod_{i \in P} p_{i}\right)}+(-1)^{|P|+l_{1}} \frac{\varphi\left(n / p_{t}\right)}{\varphi\left(p_{s}^{l_{1}} \prod_{i \in P} p_{i}\right)} \\
& =(-1)^{|P|} \frac{(-1)^{l_{2}} \varphi\left(n / p_{s}\right) \varphi\left(p_{s}^{l_{1}}\right)+(-1)^{l_{1}} \varphi\left(n / p_{t}\right) \varphi\left(p_{t}^{l_{2}}\right)}{\varphi\left(p_{s}^{l_{1}}\right) \varphi\left(p_{t}^{l_{2}}\right) \varphi\left(\prod_{i \in P} p_{i}\right)} .
\end{aligned}
$$

If $l_{1}=l_{2}$ then

$$
\left|\lambda_{j}\right|=\frac{\varphi\left(n / p_{s}\right) \varphi\left(p_{s}^{l_{1}}\right)+\varphi\left(n / p_{t}\right) \varphi\left(p_{t}^{l_{2}}\right)}{\varphi\left(p_{s}^{l_{1}}\right) \varphi\left(p_{t}^{l_{2}}\right) \varphi\left(\prod_{i \in P} p_{i}\right)}
$$

while for $l_{1} \neq l_{2}$ we have

$$
\left|\lambda_{j}\right|=\frac{(-1)^{l_{2}} \varphi\left(n / p_{s}\right) \varphi\left(p_{s}^{l_{1}}\right)+(-1)^{l_{1}} \varphi\left(n / p_{t}\right) \varphi\left(p_{t}^{l_{2}}\right)}{\varphi\left(p_{s}^{l_{1}}\right) \varphi\left(p_{t}^{l_{2}}\right) \varphi\left(\prod_{i \in P} p_{i}\right)},
$$

except for $p_{s}=2, p_{t}^{2} \mid n$ and $n \in 4 \mathbb{N}+2$.

It can be noticed that the numerator of the above relation for $l_{1}=0$ and $l_{2}=1$ is reduced to

$$
\varphi\left(n / p_{t}\right) \varphi\left(p_{t}\right)-\varphi\left(n / p_{s}\right)
$$

while for $l_{1}=1$ and $l_{2}=0$ we have

$$
\varphi\left(n / p_{s}\right) \varphi\left(p_{s}\right)-\varphi\left(n / p_{t}\right)
$$

Since Euler totient function is multiplicative, for $\alpha_{s}=1$ we have

$$
\varphi(n)=\left(p_{s}-1\right) \varphi\left(n / p_{s}\right) .
$$

Therefore, if $p_{s} \| n$ and $p_{t} \| n$ the above expressions are equivalent to $\varphi(n)-\varphi\left(n / p_{s}\right)$ and $\varphi(n)-\varphi\left(n / p_{t}\right)$.

Now assume that $p_{s}^{2} \mid n$ and $p_{t}^{2} \mid n$. We may conclude that both expressions (16) and (17) are greater than zero if and only if $\left(p_{s}-1\right)\left(p_{t}-1\right)>1$. The last relation is trivially satisfied.

If $p_{s}^{2} \mid n$ and $p_{t} \| n$ then expression (16) is equivalent to $\varphi(n)-\varphi\left(n / p_{s}\right)$, which is greater than zero. Expression (17) is greater or equal to zero if and only if $\left(p_{s}-1\right)\left(p_{t}-2\right) \geq 1$. This is true, since $p_{t}>p_{s} \geq 2$.

If $p_{s} \| n$ and $p_{t}^{2} \mid n$ then expression (17) is equivalent to $\varphi(n)-\varphi\left(n / p_{t}\right)$, which is greater than zero. Expression (16) is greater or equal to zero if and only if $\left(p_{s}-2\right)\left(p_{t}-1\right) \geq 1$. This is true, only if $p_{s}>2$. Therefore, for $p_{s}=2, p_{t}^{2} \mid n$ and $n \in 4 \mathbb{N}+2$ we have that

$$
\left|\lambda_{j}\right|=\frac{\varphi(n)-\varphi\left(n / p_{t}\right) \varphi\left(p_{t}\right)}{\varphi\left(p_{s}^{l_{1}}\right) \varphi\left(p_{t}^{l_{2}}\right) \varphi\left(\prod_{i \in P} p_{i}\right)}
$$


if $l_{1}=0$ and $l_{2}=1$, while

$$
\left|\lambda_{j}\right|=\frac{\varphi(n)-\varphi\left(n / p_{t}\right)}{\varphi\left(p_{s}^{l_{1}}\right) \varphi\left(p_{t}^{l_{2}}\right) \varphi\left(\prod_{i \in P} p_{i}\right)},
$$

if $l_{1}=1$ and $l_{2}=0$.

The number of indices $j \in J_{l_{1}, l_{2}}$ with the same set $P$ in all mentioned cases is given by (14) and equals

$$
\varphi\left(p_{s}^{l_{1}}\right) \varphi\left(p_{t}^{l_{2}}\right) \varphi\left(\prod_{i \in P} p_{i}\right)
$$

Case 2. $l_{1}=2,0 \leq l_{2} \leq 1$.

According to relation (13) we have that $t_{n / p_{t}, j}=p_{s}^{2} \prod_{i \in P} p_{i}$, which further implies that $c\left(j, n / p_{t}\right)=$ 0 . Now, using relation (12) it holds that $t_{n / p_{t}, j}=p_{s} p_{t}^{l_{2}} \prod_{i \in P} p_{i}$ and thus

$$
\lambda_{j}=c\left(j, n / p_{s}\right)=(-1)^{|P|+l_{2}+1} \frac{\varphi\left(n / p_{s}\right)}{\varphi\left(p_{s}\right) \varphi\left(p_{t}^{l_{2}}\right) \varphi\left(\prod_{i \in P} p_{i}\right)} .
$$

The number of indices $j \in J_{l_{1}, l_{2}}$ with the same set $P$ is given by (14) and equals

$$
p_{s} \varphi\left(p_{s}\right) \varphi\left(p_{t}^{l_{2}}\right) \varphi\left(\prod_{i \in P} p_{i}\right)
$$

Case 3. $0 \leq l_{1} \leq 1, l_{2}=2$.

In this case we obtain symmetric expressions for $\lambda_{j}$ and the number of indices with given set $P$.

Case 4. $l_{1}=l_{2}=2$.

According to the relations (12) and (13) we have that $t_{n / p_{s}, j}=p_{t}^{2} \prod_{i \in P} p_{i}$ and $t_{n / p_{t}, j}=p_{s}^{2} \prod_{i \in P} p_{i}$, which further implies $\lambda_{j}=c\left(j, n / p_{s}\right)=c\left(j, n / p_{t}\right)=0$.

By summarizing all formulas in mention cases, the energy of $X_{n}\left(p_{s}, p_{t}\right)$ is given by

$$
\begin{aligned}
E\left(X_{n}\left(p_{s}, p_{t}\right)\right)= & \sum_{j=0}^{n-1}\left|\lambda_{j}\right| \\
= & \sum_{P \subseteq\{1,2, \ldots, k\} \backslash\{s, t\}}\left(\left(\varphi\left(n / p_{s}\right)+\varphi\left(n / p_{t}\right)\right)+\left(\varphi\left(n / p_{s}\right) \varphi\left(p_{s}\right)+\varphi\left(n / p_{t}\right) \varphi\left(p_{t}\right)\right)\right. \\
& +\left(\varphi\left(n / p_{t}\right) \varphi\left(p_{t}\right)-\varphi\left(n / p_{s}\right)\right)+\left(\varphi\left(n / p_{s}\right) \varphi\left(p_{s}\right)-\varphi\left(n / p_{t}\right)\right) \\
& +2 \varphi\left(n / p_{s}\right) p_{s} \\
& \left.+2 \varphi\left(n / p_{t}\right) p_{t}\right)
\end{aligned}
$$

If $\alpha_{s}=\alpha_{t}=1$ then only nonempty sets are $J_{0,0}, J_{0,1}, J_{1,0}$ and $J_{1,1}$. Thus, the relation (20) becomes

$$
\begin{aligned}
E\left(X_{n}\left(p_{s}, p_{t}\right)\right)= & 2^{k-2} \cdot\left(\left(\varphi\left(n / p_{s}\right)+\varphi\left(n / p_{t}\right)\right)+\left(\varphi\left(n / p_{s}\right) \varphi\left(p_{s}\right)+\varphi\left(n / p_{t}\right) \varphi\left(p_{t}\right)\right)\right. \\
& +\left(\varphi\left(n / p_{t}\right) \varphi\left(p_{t}\right)-\varphi\left(n / p_{s}\right)\right)+\left(\varphi\left(n / p_{s}\right) \varphi\left(p_{s}\right)-\varphi\left(n / p_{t}\right)\right) \\
= & 2^{k-2}(4 \varphi(n))=2^{k} \varphi(n) .
\end{aligned}
$$

If $\alpha_{s}=1, \alpha_{t}>1$ and $p_{s} \neq 2$ then $J_{2,0}, J_{2,1}$ and $J_{2,2}$ are the empty sets. Also, for $\alpha_{t}>1$ we have

$$
\varphi(n)=\left(p_{t}-1\right) p_{t}^{\alpha_{t}-1} \varphi\left(n / p_{t}^{\alpha_{t}}\right)=p_{t} \varphi\left(p_{t}^{\alpha_{t}-1}\right) \varphi\left(n / p_{t}^{\alpha_{t}}\right)=p_{t} \varphi\left(n / p_{t}\right)>\left(p_{t}-1\right) \varphi\left(n / p_{t}\right) .
$$

Therefore, from the relation (20) follows

$$
E\left(X_{n}\left(p_{s}, p_{t}\right)\right)=2^{k-2} \cdot\left(2\left(\varphi(n)+\varphi\left(n / p_{t}\right) \varphi\left(p_{t}\right)\right)+2 \varphi\left(n / p_{t}\right) p_{t}\right)=2^{k-1}\left(2 \varphi(n)+\varphi\left(n / p_{t}\right) \varphi\left(p_{t}\right)\right) .
$$


If $\alpha_{s}=1, \alpha_{t}>1$ and $p_{s}=2$, according to relations (18) and (19) the energy is equal to

$$
\begin{aligned}
E\left(X_{n}\left(p_{s}, p_{t}\right)\right)= & 2^{k-2} \cdot\left(\varphi\left(n / p_{s}\right)+\varphi\left(n / p_{t}\right)\right)+\left(\varphi\left(n / p_{s}\right) \varphi\left(p_{s}\right)+\varphi\left(n / p_{t}\right) \varphi\left(p_{t}\right)\right) \\
& +\left(\varphi(n)-\varphi\left(n / p_{t}\right) \varphi\left(p_{t}\right)\right)+\left(\varphi(n)-\varphi\left(n / p_{t}\right)\right) \\
& +2 p_{t} \varphi\left(p_{t}\right) \\
= & 2^{k-1}\left(2 \varphi(n)+\varphi\left(n / p_{t}\right) p_{t}\right)=3 \cdot 2^{k-1} \varphi(n) .
\end{aligned}
$$

If $\alpha_{s}>1$ and $\alpha_{t}=1$, we have similar equation as in the previous case:

$$
E\left(X_{n}\left(p_{s}, p_{t}\right)\right)=2^{k-1}\left(2 \varphi(n)+\varphi\left(n / p_{s}\right) \varphi\left(p_{s}\right)\right) .
$$

If $\alpha_{s}>1$ and $\alpha_{t}>1$ then all sets $J_{l_{1}, l_{2}}$, for $0 \leq l_{1}, l_{2} \leq 2$ are nonempty and thus the energy is equal to

$$
\begin{aligned}
E\left(X_{n}\left(p_{s}, p_{t}\right)\right) & =2^{k-2} \cdot\left(2\left(\varphi\left(n / p_{s}\right) \varphi\left(p_{s}\right)+\varphi\left(n / p_{t}\right) \varphi\left(p_{t}\right)\right)+2 \varphi\left(n / p_{s}\right) p_{s}+2 \varphi\left(n / p_{t}\right) p_{t}\right) \\
& =2^{k-1}\left(2 \varphi(n)+\varphi\left(n / p_{s}\right) p_{s}+\varphi\left(n / p_{t}\right) \varphi\left(p_{t}\right)\right) .
\end{aligned}
$$

This completes the proof.

\section{Classes of non-cospectral graphs with equal energy}

Let $n=p_{1} p_{2} \ldots p_{s} p_{s+1}^{\alpha_{s+1}} \ldots p_{k}^{\alpha_{k}}$ be a prime factorization of $n$, where $\alpha_{i} \geq 2$ for $s+1 \leq i \leq k$. Using the result of Theorem (4.2) we see that the energy of integral circulant graph $X_{n}\left(p_{i}, p_{j}\right)$ does not depend on the choice of $p_{i}$ and $p_{j}$, if $p_{i}, p_{j} \| n$. Also, the same conclusion can be derived if we consider the graphs $X_{n}\left(2, p_{j}\right)$ for $\alpha_{j} \geq 2$ and $n \in 4 \mathbb{N}+2$.

Since the order of the graph $X_{n}\left(p_{i}, p_{j}\right)$ is equal to $\varphi\left(n / p_{i}\right)+\varphi\left(n / p_{j}\right)$, which is at the same time the largest eigenvalues also, we can construct at least $s+1$ non-cospectral regular $n$-vertex hyperenergetic graphs,

$$
X_{n}(1), X_{n}\left(p_{1}, p_{2}\right), X_{n}\left(p_{1}, p_{3}\right), \ldots, X_{n}\left(p_{1}, p_{s}\right),
$$

with equal energy. Similarly, we obtain the second class of $k-s$ non-cospectral graphs with equal energy.

$$
X_{n}\left(2, p_{s+1}\right), X_{n}\left(2, p_{s+2}\right), \ldots, X_{n}\left(2, p_{k}\right),
$$

Moreover, we can consider a square-free number $n=p_{1} p_{2} \cdot \ldots \cdot p_{k}$ and prove that the following $\left(\begin{array}{l}k \\ 2\end{array}\right)$ graphs

$$
X_{n}\left(p_{1}, p_{2}\right), X_{n}\left(p_{1}, p_{3}\right), \ldots, X_{n}\left(p_{k-1}, p_{k}\right),
$$

are non-cospectral.

Consider the integral circulant graph $X_{n}\left(p_{i}, p_{j}\right)$. The largest eigenvalue and the degree of $X_{n}\left(p_{i}, p_{j}\right)$ is $\varphi\left(n / p_{i}\right)+\varphi\left(n / p_{j}\right)$. According to the proof of Theorem 4.2 from [22], the second largest value among $\left|\lambda_{1}\right|,\left|\lambda_{2}\right|, \ldots,\left|\lambda_{n-1}\right|$ equals

$$
\begin{aligned}
s\left(X_{n}\left(p_{i}, p_{j}\right)\right) & =\max \left\{\frac{\varphi\left(\frac{n}{p_{i}}\right)+\varphi\left(\frac{n}{p_{j}}\right)}{\varphi(p)}, \frac{\varphi(n)-\varphi\left(\frac{n}{p_{i}}\right)}{\varphi\left(p_{j}\right)}, \frac{\varphi(n)-\varphi\left(\frac{n}{p_{j}}\right)}{\varphi\left(p_{i}\right)}, \frac{2 \varphi(n)}{\varphi\left(p_{i} p_{j}\right)}\right\} \\
& =\varphi\left(\frac{n}{p_{i} p_{j}}\right) \cdot \max \left\{\frac{p_{i}+p_{j}-2}{\varphi\left(p_{i j}\right)}, p_{i}-2, p_{j}-2,2\right\},
\end{aligned}
$$

where $p_{i j}$ denotes the smallest prime number dividing $\frac{n}{p_{i} p_{j}}$. 
Assume that graphs $X_{n}\left(p_{i}, p_{j}\right)$ and $X_{n}\left(p_{r}, p_{q}\right)$ are cospectral, with $p_{j}>p_{i}$ and $p_{q}>p_{r}$. Furthermore, assume that $p_{i}>p_{r}$.

Case 1. $p_{i}>3$ and $p_{r}>3$.

From $p_{j}>p_{i}>3$ it easily follows that

$$
s\left(X_{n}\left(p_{i}, p_{j}\right)\right)=\varphi\left(\frac{n}{p_{i} p_{j}}\right) \cdot\left(p_{j}-2\right) .
$$

By equating the largest eigenvalues of these graphs and the values $s\left(X_{n}\left(p_{i}, p_{j}\right)\right)$ and $s\left(X_{n}\left(p_{r}, p_{q}\right)\right)$, it follows

$$
\begin{aligned}
\varphi\left(p_{r} p_{q}\right) \cdot\left(p_{i}+p_{j}-2\right) & =\varphi\left(p_{i} p_{j}\right) \cdot\left(p_{r}+p_{q}-2\right) \\
\varphi\left(p_{r} p_{q}\right) \cdot\left(p_{j}-2\right) & =\varphi\left(p_{i} p_{j}\right) \cdot\left(p_{q}-2\right) .
\end{aligned}
$$

Notice that we used the multiplicative property of the Euler function.

By subtraction, we get

$$
\left(p_{r}-1\right)\left(p_{q}-1\right) \cdot p_{i}=\left(p_{i}-1\right)\left(p_{j}-1\right) \cdot p_{r} .
$$

Assume without loss of generality that $p_{i}<p_{r}$. It follows that $p_{i} \mid p_{j}-1$ and $p_{r} \mid p_{q}-1$. Since $p_{i} \mid \varphi\left(p_{j}\right)$ and $p_{r} \mid \varphi\left(p_{q}\right)$, from the relation (26), we conclude that $p_{i} \mid \varphi\left(p_{r} p_{q}\right)$ and $p_{r} \mid \varphi\left(p_{i} p_{j}\right)$. Since $p_{i}<p_{r}$, we have $p_{r} \mid p_{j}-1$ and from the relation (28) it holds that $p_{r}^{2} \mid p_{q}-1$. Similarly, from the relation (26) it follows that $p_{r}^{2} \mid p_{j}-1$ and again according to (26) $p_{r}^{3} \mid p_{q}-1$ holds. Using infinite descent, we get that four-tuple $\left(p_{i}, p_{j}, p_{r}, p_{q}\right)$ does not exist.

Case 2. $p_{i}>3$ and $p_{r}=3$.

We distinguish two cases depending on the values of $p_{r q}$. Let $p_{r q}=2$. Then, according to the relation (25) we have that

$$
s\left(X_{n}\left(p_{r}, p_{q}\right)\right)=\varphi\left(\frac{n}{3 p_{q}}\right) \cdot\left(p_{q}+1\right) .
$$

By equating the largest eigenvalues of these graphs and the values $s\left(X_{n}\left(p_{i}, p_{j}\right)\right)$ and $s\left(X_{n}\left(p_{r}, p_{q}\right)\right)$, it follows

$$
\begin{gathered}
\varphi\left(3 p_{q}\right) \cdot\left(p_{i}+p_{j}-2\right)=\varphi\left(p_{i} p_{j}\right) \cdot\left(p_{q}+1\right) \\
\varphi\left(3 p_{q}\right) \cdot\left(p_{j}-2\right)=\varphi\left(p_{i} p_{j}\right) \cdot\left(p_{q}+1\right) .
\end{gathered}
$$

The last two equation hold only if $p_{i}+p_{j}-2=p_{j}-2$ which a contradiction.

Let $p_{r q}>2$. Since $p_{q}>p_{r}=3$ and therefore $p_{q} \geq 5$, we have that

$$
p_{q}-2 \geq \frac{p_{q}+1}{2} \geq \frac{p_{q}+1}{\varphi\left(p_{r q}\right)} .
$$

From the last relation we conclude that

$$
s\left(X_{n}\left(p_{r}, p_{q}\right)\right)=\varphi\left(\frac{n}{3 p_{q}}\right) \cdot \frac{p_{q}+1}{\varphi\left(p_{r q}\right)} .
$$

By equating the largest eigenvalues of these graphs and the values $s\left(X_{n}\left(p_{i}, p_{j}\right)\right)$ and $s\left(X_{n}\left(p_{r}, p_{q}\right)\right)$, it follows

$$
\begin{gathered}
\varphi\left(3 p_{q}\right) \cdot\left(p_{i}+p_{j}-2\right)=\varphi\left(p_{i} p_{j}\right) \cdot\left(p_{q}+1\right) \\
\varphi\left(3 p_{q}\right) \cdot \varphi\left(p_{r q}\right) \cdot\left(p_{j}-2\right)=\varphi\left(p_{i} p_{j}\right) \cdot\left(p_{q}+1\right) .
\end{gathered}
$$

From the last relations we see that $p_{i}+p_{j}-2=\varphi\left(p_{r q}\right) \cdot\left(p_{j}-2\right)$ holds. Next, it holds that $p_{i} \leq p_{j}-2$, which further implies $\varphi\left(p_{r q}\right) \cdot\left(p_{j}-2\right) \leq 2\left(p_{j}-2\right)$. But that is only the case if $\varphi\left(p_{r q}\right) \leq 2$ or equivalently $p_{r q} \leq 3$, which is a contradiction. 
Case 3. $p_{i}>3$ and $p_{r}=2$.

We distinguish two cases depending on the values of $p_{q}$.

Let $p_{q}=3$. From the relation (25) it can be concluded that

$$
s\left(X_{n}\left(p_{r}, p_{q}\right)\right)=2 \cdot \varphi\left(\frac{n}{6}\right) .
$$

By equating the largest eigenvalues of these graphs and the values $s\left(X_{n}\left(p_{i}, p_{j}\right)\right)$ and $s\left(X_{n}\left(p_{r}, p_{q}\right)\right)$, it follows

$$
\begin{gathered}
\varphi(6) \cdot\left(p_{i}+p_{j}-2\right)=3 \cdot \varphi\left(p_{i} p_{j}\right) \\
\varphi(6) \cdot\left(p_{j}-2\right)=2 \cdot \varphi\left(p_{i} p_{j}\right)
\end{gathered}
$$

By subtraction, we get

$$
\varphi(6) \cdot p_{i}=\varphi\left(p_{i} p_{j}\right)=\left(p_{i}-1\right)\left(p_{j}-1\right) .
$$

From the last relation it holds that $p_{i} \mid p_{j}-1$ and combining with the relation (29)) we obtain that $p_{i} \mid p_{j}-2$. This is a contradiction, since $p_{j}-2$ and $p_{j}-2$ are relatively prime.

Let $p_{q}>3$. Since the following inequality holds

$$
p_{q}-2 \geq \frac{p_{q}}{2} \geq \frac{p_{q}}{\varphi\left(p_{r q}\right)}
$$

we have

$$
s\left(X_{n}\left(p_{r}, p_{q}\right)\right)=\varphi\left(\frac{n}{3 p_{q}}\right) \cdot\left(p_{q}-2\right) .
$$

Now, this case is reduced to the equations (26) and (27) from Case 1, where we obtained a contradiction.

Case 4. $p_{i}=3$ and $p_{r}=2$.

Since $p_{q} \neq p_{i}$ we have $p_{q} \geq 5$, which further implies $\left.\max \left\{p_{q} / \varphi\left(p_{r q}\right), p_{q}-2,2\right)\right\}=p_{q}-2$. Therefore, it holds that

$$
s\left(X_{n}\left(p_{r}, p_{q}\right)\right)=\varphi\left(\frac{n}{2 p_{q}}\right)\left(p_{q}-2\right) .
$$

Moreover, as $p_{j} \neq p_{r}$ and $p_{r}=2$, we obtain $p_{i j}=2$. Thus, we conclude

$$
\left.\max \left\{\frac{p_{j}+1}{\varphi\left(p_{i j}\right)}, 1, p_{j}-2,2\right)\right\}=p_{j}+1
$$

and

$$
s\left(X_{n}\left(p_{i}, p_{j}\right)\right)=\varphi\left(\frac{n}{3 p_{j}}\right)\left(p_{j}+1\right) .
$$

By equating the largest eigenvalues of these graphs and the values $s\left(X_{n}\left(p_{i}, p_{j}\right)\right)$ and $s\left(X_{n}\left(p_{r}, p_{q}\right)\right)$, it follows

$$
\begin{gathered}
\varphi\left(2 p_{q}\right) \cdot\left(p_{j}+1\right)=\varphi\left(3 p_{j}\right) \cdot p_{q} \\
\varphi\left(2 p_{q}\right) \cdot\left(p_{j}+1\right)=\varphi\left(3 p_{j}\right) \cdot\left(p_{q}-2\right) .
\end{gathered}
$$

From the previous relations we trivially get that four-tuple $\left(p_{i}, p_{j}, p_{r}, p_{q}\right)$ does not exist in this case.

This way we actually prove that two cospectral integral circulant graphs $\operatorname{ICG}_{n}\left(D_{1}\right)$ and $\operatorname{ICG}_{n}\left(D_{2}\right)$ must be isomorphic i.e. $D_{1}=D_{2}$, for a square-free number $n$ and two-element divisor sets $D_{1}$ and $D_{2}$ containing prime divisors. Therefore, we support conjecture proposed by So [34], that two graphs $\mathrm{ICG}_{n}\left(D_{1}\right)$ and $\mathrm{ICG}_{n}\left(D_{2}\right)$ are cospectral if and only if $D_{1}=D_{2}$. The conjecture was only proven for the trivial cases where $n$ being square-free and product of two primes. Our result is obviously one form of generalization. 


\section{Concluding remarks}

In this paper we focus on some global characteristics of the energy of integral circulant graphs such as energy modulo four and existence of non-cospectral graphs classes with equal energy. We also find explicit formulas for the energy of $\operatorname{ICG}_{n}(D)$ classes with two-element set $D$. In contrast to [22], the calculation of these formulas require extensive discussion in many different cases. Some further generalizations on this topic would require much more case analysis. The examples of such generalizations are calculating the energy of the graphs with three or more divisors, graphs with square-free orders etc. The general problem of calculating the energy of $\operatorname{ICG}_{n}(D)$ graphs seems very difficult, since as we increase the number of divisors in $D$ we have more sign changes in Ramanujan functions $c(n, i)$.

For the further research we also propose some new general characteristics of the energy such as studying minimal and maximal energies for a given integral circulant graph, and characterizing the extremal graphs. We will use the following nice result from [18, 37.

Theorem 6.1 Let $G$ be a regular graph on $n$ vertices of degree $r>0$. Then

$$
E(G) \geq n,
$$

with equality if and only if every component of $G$ is isomorphic to the complete bipartite graph $K_{r, r}$.

The proof is based on the estimation

$$
E(G) \geq \frac{M_{2}^{2}}{\sqrt{M_{2} M_{4}}}
$$

where $M_{2}=2 m$ and $M_{4}$ are spectral moments of graph $G$, defined as

$$
M_{k}=\sum_{i=1}^{n} \lambda_{i}^{k} .
$$

The fourth moment is equal to $M_{4}=8 q-2 m+2 \sum_{v \in V} d e g^{2}(v)$, where $q$ is the number of quadrangles in $G$.

Let $n$ be even number and assume that $\operatorname{ICG}_{n}\left(D^{*}\right)$ is isomorphic to $K_{n / 2, n / 2}$. The present authors in [6] proved the following

Theorem 6.2 Let $d_{1}, d_{2}, \ldots, d_{k}$ be divisors of $n$ such that the greatest common divisor $\operatorname{gcd}\left(d_{1}, d_{2}, \ldots, d_{k}\right)$ equals $d$. Then the graph $\operatorname{ICG}_{n}\left(d_{1}, d_{2}, \ldots, d_{k}\right)$ has exactly $d$ connected components isomorphic to $\mathrm{ICG}_{n / d}\left(\frac{d_{1}}{d}, \frac{d_{2}}{d}, \ldots, \frac{d_{k}}{d}\right)$.

In this case the complement of $\mathrm{ICG}_{n}\left(D^{*}\right)$, denoted by $\mathrm{ICG}_{n}(\bar{D})$, must contain exactly two connected components that are cliques, and for $\bar{D}=\left\{d_{1}, d_{2}, \ldots, d_{k}\right\}$ we have $\operatorname{gcd}\left(d_{1}, d_{2}, \ldots, d_{k}\right)=2$ and $\mathrm{ICG}_{n / 2}\left(\frac{d_{1}}{2}, \frac{d_{2}}{2}, \ldots, \frac{d_{k}}{2}\right)$ is isomorphic to a complete graph $K_{n / 2}$. It simply follows that the set $\bar{D}$ must contain all even divisors of $n$ and therefore $D^{*}$ is the set of all odd divisors of $n$. Therefore, the degree of $\operatorname{ICG}_{n}\left(D^{*}\right)$ is equal to $\frac{n}{2}=\sum_{d \in D^{*}} \varphi\left(\frac{n}{d}\right)$ and $\operatorname{ICG}_{n}\left(D^{*}\right)$ is isomorphic to a complete bipartite graph $K_{n / 2, n / 2}$. Recall that the spectra of the complete bipartite graph $K_{m, n}$ consists of $\sqrt{m n},-\sqrt{m n}$ and 0 with multiplicity $n-2$. It follows that $\left|\lambda_{n / 2}\right|=\left|\lambda_{0}\right|=\frac{n}{2}$ and for $k \neq 0, \frac{n}{2}$ we have the following nice identity

$$
\begin{aligned}
\lambda_{k} & =\sum_{d \mid n, d \text { odd }} c(k, d) \\
& =\sum_{d \mid n, d \text { odd }} \mu\left(\frac{d}{\operatorname{gcd}(k, d)}\right) \cdot \frac{\varphi(d)}{\varphi\left(\frac{d}{\operatorname{gcd}(k, d)}\right)} \\
& =0 .
\end{aligned}
$$


Using computer search, for odd $n$ the minimum is $2 n\left(1-\frac{1}{p}\right)$, where $p$ is the smallest prime dividing $n$. The extremal integral circulant graph contains all divisors of $n$ that are not divisible by $p$ (and the complement of such graph is composed of $p$ cliques). We leave this observation as a conjecture.

Acknowledgement. The authors gratefully acknowledge support from Research projects 174010 and 174033 of the Serbian Ministry of Science.

\section{References}

[1] A. Ahmadi, R. Belk, C. Tamon and C. Wendler, On mixing of continuous time quantum walks on some circulant graphs, Quant. Inform. Comput. 3 (2003) 611-618.

[2] R.J. Angeles-Canul, R.M. Norton, M.C. Opperman, C.C. Paribello, M.C. Russell, C. Tamonk, Perfect state transfer, integral ciculants and join of graphs, Quant. Inform. Comput. 10 (2010) 325-342.

[3] R.J. Angeles-Canul, R.M. Norton, M.C. Opperman, C.C. Paribello, M.C. Russell, C. Tamonk, Quantum perfect state transfer on weighted join graphs, Int. J. Quantum Inf. 7 (2009), 1429-1445.

[4] S. Akbari, F. Moazami, S. Zare, Kneser Graphs and their Complements are Hyperenergetic, MATCH Commun. Math. Comput. Chem. 61 (2009), 361-368.

[5] R. B. Bapat, S. Pati, Energy of a graph is never an odd integer, Bull. Kerala Math. Assoc. 1 (2004) 129-132.

[6] M. Bašić, A. Ilić, On the clique number of integral circulant graphs, Appl. Math. Lett. 22 (2009) 1406-1411.

[7] M. Bašić, M. Petković, D. Stevanović, Perfect state transfer in integral circulant graphs, Appl. Math. Lett. 22 (2009) 1117-1121.

[8] M. Bašić, Characterization of circulant graphs having perfect state transfer, manuscript, 2010.

[9] S. Blackburn, I. Shparlinski, On the average energy of circulant graphs, Linear Algebra Appl. 428 (2008), 1956-1963.

[10] A. S. Bonifácio, C. T. M. Vinagre, N. M. M. de Abreu, Constructing pairs of equienergetic and non-cospectral graphs, Appl. Math. Lett. 21 (2008), 338-341.

[11] V. Brankov, D. Stevanović, I. Gutman, Equienergetic chemical trees, J. Serb. Chem. Soc. 69 (2004), 549-553.

[12] M. Christandl, N. Datta, T.C. Dorlas, A. Ekert, A. Kay, and A.J. Landahl, Perfect transfer of arbitrary states in quantum spin networks, Phys. Rev. A 71:032312, 2005.

[13] P. J. Davis, Circulant matrices, Pure and Applied Mathematics, John Wiley \& Sons, New YorkChichester-Brisbane, 1979.

[14] C. D. Godsil, Periodic Graphs, arXiv:0806.2074v1 [math.CO], 12 Jun 2008.

[15] I. Gutman, The energy of a graph, Ber. Math. Stat. Sekt. Forschungszent. Graz 103 (1978) 1-22.

[16] I. Gutman, The energy of a graph: Old and new results, in: A. Betten, A. Kohnert, R. Laue, A. Wassermann (Eds.), Algebraic Combinatorics and Applications, Springer-Verlag, Berlin, 2001, pp. $196-211$. 
[17] I. Gutman, Hyperenergetic molecular graphs, J. Serb. Chem. Soc. 64 (1999) 199-205.

[18] I. Gutman, S. Z. Firoozabadi, J. A. de la Peña, J. Rada, On the energy of regular graphs, MATCH Commun. Math. Comput. Chem. 57 (2007) 435-442.

[19] I. Gutman, X. Li, J. Zhang, Graph energy, in: M. Dehmer, F. Emmert-Streib (Eds.), Analysis of Complex Networks. From Biology to Linguistics, Wiley-VCH, Weinheim, 2009, pp. 145-174.

[20] G. H. Hardy, E. M. Wright, An Introduction to the Theory of Numbers, Fifth edition, Oxford University Press, New York, 1980.

[21] F. K. Hwang, A survey on multi-loop networks, Theoretical Computer Science 299 (2003) 107-121.

[22] A. Ilić, The energy of unitary Cayley graphs, Linear Algebra Appl. 431 (2009) 1881-1889.

[23] A. Ilić, M. Bašić, On the chromatic number of integral circulant graphs, Comput. Math. Appl. 60 (2010) 144-150.

[24] A. Ilić, M. Bašić, I. Gutman, Triply Equienergetic Graphs, MATCH Commun. Math. Comput. Chem. 64 (2010) 189-200.

[25] A. Ilić, Distance spectra and distance energy of integral circulant graphs, Linear Algebra Appl. 433 (2010) 1005-1014.

[26] W. Klotz, T. Sander, Some properties of unitary Cayley graphs, Electron. J. Combin. 14 (2007) \#R45

[27] J. Liu, B. Liu, E-L equienergetic graphs, MATCH Commun. Math. Comput. Chem. 66 (2011) 971-976.

[28] O. Miljković, B. Furtula, S. Radenković, I. Gutman, Equienergetic and almost-equienergetic trees, MATCH Commun. Math. Comput. Chem. 61 (2009) 451-461.

[29] S. Pirzada, I. Gutman, Energy of a graph is never the square root of an odd integer, Appl. Anal. Discrete Math. 2 (2008) 118-121.

[30] H. S. Ramane, H. B. Walikar, Construction of equienergetic graphs, MATCH Commun. Math. Comput. Chem. 57 (2007), 203-210.

[31] H. N. Ramaswamy, C. R. Veena, On the Energy of Unitary Cayley Graphs, Electron. J. Combin. 16 (2007) \#N24

[32] O. Rojo, L. Medina, Constructing graphs with energy $\sqrt{r} E(G)$ where $G$ is a bipartite graph, MATCH Commun. Math. Comput. Chem. 62 (2009) 465-472.

[33] N. Saxena, S. Severini, I. Shparlinski, Parameters of integral circulant graphs and periodic quantum dynamics, Int. J. Quant. Inf. 5 (2007), 417-430.

[34] W. So, Integral circulant graphs, Discrete Math. 306 (2006) 153-158.

[35] W. So, Remarks on some graphs with large number of edges, MATCH Commun. Math. Comput. Chem. 61 (2009), 351-359.

[36] I. Stanković, M. Milošević, D. Stevanović, Small and not so small equienergetic graphs, MATCH Commun. Math. Comput. Chem. 61 (2009) 443-450.

[37] B. Zhou, I. Gutman, J. A. de la Peña, J. Rada, L. Mendoza, On spectral moments and energy of graphs, MATCH Commun. Math. Comput. Chem. 57 (2007) 183-191. 
[38] http://en.wikipedia.org/wiki/Ramanujan's_sum 